\title{
Electromagnetic Fields and Spatial Memory
}

\begin{abstract}
Electromagnetic fields seriously damage the physical and psychological health. Reports on increasing changes in cognitive and behavioral functions in human and animal models have proposed electromagnetic fields as environmental contaminants. This study tries to review the effects of exposure to the electromagnetic fields on spatial memory as one of the higher brain processes of animal models. It is suggested that brain changes in neurochemical and neuroanatomic structures after exposure to the electromagnetic radiation will change the behavioral responses in memory tests.
\end{abstract}

Keywords: animal models, electromagnetic fields, memory
Volume I Issue 3 - 2015

\author{
Elham Foroozandeh \\ Department of Psychology, Islamic Azad University, Iran
}

Correspondence: Elham Foroozandeh, Department of Psychology, Islamic Azad University, Iran, Tel 989133004695, Email elham_for@yahoo.com

Received: April 27, 2015 | Published: August 29, 2015
Abbreviations: EMF, electromagnetic fields; ELF-EMF, extremely low frequency of electromagnetic fields

\section{Introduction}

After exposure to the EMFs increased risk of Alzheimer's diseases and central nervous system disorders are reported in human. ${ }^{1}$ In animal models depending on the fields characters (electric, magnetic, or electromagnetic), static or changing frequency (low, medium, high), and also the modulation of waves (pulsed waves or constant waves), strength and power density of the field, and duration of exposure to the field different consequences are proposed like reduction of natural defense system, level of health elimination and reproductive problems. ${ }^{2,3}$ The purpose of this study is to investigate spatial memory of exposed animal to EMFs in the laboratory controlled situation.

Morris water maze is one of the best tools to assess spatial memory in rats. It is reported that a 20 minutes exposure to $8 \mathrm{mT}$ field after training in water maze that rats in a water pool with $140 \mathrm{~cm}$ diameter and $50 \mathrm{~cm}$ depth learn to find the platform, would disrupt the consolidation of spatial information, whereas the $2 \mathrm{mT}$ field won't cause such damages however there is no difference in comparison between the swimming speed and the movement. ${ }^{4}$ Exposure for $60 \mathrm{~min}$ before training rats in water maze to $60 \mathrm{HZ}, 1 \mathrm{mT}$ field is harmful to their spatial memory ${ }^{5}$ and it is stated that exposure to the field of $2 \mathrm{mT}$ (not less than 2) for $60 \mathrm{~min}$ have decreased effect on cholinergic activities in the frontal cortex and hippocampus, and the same effect can be observed in longer duration (1.5 and $3 \mathrm{~h}$ ) of exposure to the fields of lower intensity $(0.5,1,1.5 \mathrm{mT}){ }^{6}$ Since the cholinergic system have an important role in memory processes, any reduction of its activities could impair the memory process. ${ }^{7,8}$

The fields of 200 to $500 \mathrm{nT}$ and exposure for $60 \mathrm{~min}$ before training in the radial maze impaired spatial memory whereas rats exposure to the fields before testing phase just reduce the time of the task responses. ${ }^{9}$ In a higher density and longer time exposure to an $8 \mathrm{mT}, 50 \mathrm{~Hz}$ EMF for $4 \mathrm{~h}$ has devastating effects on memory consolidation in male and female mice Using passive avoidance task. ${ }^{10}$ The researchers reported that $60 \mathrm{~min}$ exposure to electromagnetic fields of $50 \mathrm{HZ}$ with $1 \mathrm{mT}$ intensity caused reduction in cognitive function, memory and recognition. However, there are contrary results in the same area of research. ${ }^{11}$ It is reported that there was no change in memory of rats after being in the field of $0.75 \mathrm{mT}$ for 45 minutes. Although this exposure leads to reduction of learning speed of the rats, ultimately they learn the task as well as the control group. ${ }^{12}$ Using a circular coil with an inner diameter of $8 \mathrm{~cm}$ with copper wire a 7.5MT ELF-EMF was created and it is stated that short term applied magnetic field $(50 \mathrm{HZ}$ and 7.5MT)is ineffective on the rat's T-maze skill and probably it has no effect on the spatial memory process. ${ }^{13}$ It is clear that some of these inconsistencies may be due to the differences of intensity of the field exposure and the type of the task that should be considered in any study. The researchers, who believe in the effect of field exposure on cognitive functions and also on strengthening learned information, specify the necessity of RNA and protein synthesis. ${ }^{14}$ Electromagnetic field effects on gene expression, the final three dimensional structures of protein and DNA so it can be inferred that the destructive effect of electromagnetic field indicate itself through the synthesis of protein and gene expression. ${ }^{4}$

\section{Discussion}

ELFs often have devastating effect on cognitive processes of the laboratory models. Some parts of the brain (hippocampus neurons and basal forebrain) are relatively vulnerable and a very low frequency of electromagnetic field can reduce cholinergic activity. ${ }^{6}$ Although some studies refer to the positive effect of exposure to electromagnetic fields and waves on memory, or these kinds of exposure have no effect on cognitive process, it is necessary to control these contaminants with attention to the strong evidence that the negative effects of ELFs are as one of the environmental contaminants. Unlike many other types of biological contaminants, electromagnetic fields and waves, in terms of being invisible, may be less than other similar subjects such as solid wastes, wastewater, air and water pollution have been considered, But it seems that electromagnetic fields and waves have psychologically longer devastating effects.

\section{Conclusion}

As a result it can be stated that there are doubts about the controlled results of the animals and laboratory models that implementation study in human subjects might remove them. Such a process requires interdisciplinary research in the areas of toxicology and cognitive psychology.

\section{Acknowledgements}

None. 


\section{Conflict of interest}

Author has no conflicts of interest.

\section{References}

1. Anders A, Green A, Kheifets L, et al. Epidemiology of health effects of radiofrequency exposure. Environ Health Perspect. 2004;112(7):17411754.

2. Gholampour M, Karatian SI, Shekari L, et al. The effect of contaminants of phone antennas electromagnetic waves on environment. The forth conference of environmental engineering, Iran; 2001.

3. Otto M, Von Muhlendahl KE. Electromagnetic fields (EMF) do they play a role in children's environmental health $(\mathrm{CEH})$ ? Int J Hyg Environ Health. 2007;210(5):635-644.

4. Jadidi M, Firoozabadi SM, Rashidy-Pour A, et al. Acute exposure to a $50 \mathrm{~Hz}$ magnetic field impairs consolidation of spatial memory in rats. Neurobiol Learn Mem. 2007;88(4):387-392.

5. Lai H, Carino MA, Ushijima I. Acute exposure to a $60 \mathrm{~Hz}$ magnetic field affects rats water-maze performance. Bio electromagnetics. 1998;19(2):117-122.

6. Lai H, Carino M. $60 \mathrm{~Hz}$ magnetic fields and central cholinergic activity Effects of exposure intensity and duration. Bio electromagnetics. 1999;20(5):284-289.

7. Whishaw IQ. Dissociating performance and learning deficits on spatial navigation tasks in rats subjected to cholinergic blockade. Brain Res Bull. 1989;23(4-5):347-358.
8. Whishaw IQ, Tomie JA. Cholinergic receptor blockade produces impairments in sensory subsystem for place navigation in the rat:evidence from sensory, motor, and acquisition tests in a swimming pool. Behav Neurosci. 1987;101(5):603-616.

9. McKay BE, Persinger MA. Application timing of complex magnetic fields delineates windows of post-training-pretesting vulnerability for spatial and motivational behaviors in rats. Int J Neurosci. 2000;103(14):69-77.

10. Foroozandeh E, Derakhshan-Barjoei P, Jadidi M. Toxic effects of $50 \mathrm{~Hz}$ electromagnetic field on memory consolidation in male and female mice. Toxicol Ind Health. 2013;29(3):293-299.

11. Kurokawa Y, Nitta H, Imai H, et al. No influence of short-term exposure to $50-\mathrm{Hz}$ magnetic fields on cognitive performance function in human. Int Arch Occup Environ Health. 2003;76(6):437-442.

12. Sienkiewicz ZJ, Haylock RG, Saunders RD. Deficits in spatial learning after exposure of mice to a $50 \mathrm{~Hz}$ magnetic field. Bio electromagnetics. 1998;19(2):79-84.

13. Firuzabadi SMP, Jadidi M, Vafaie AA. The effect of electromagnetic field with 7.5MT on short term spatial learning of rats. Razi Journal of Medical Sciences. 2006;(13):75-76.

14. Mc Gaugh JL. Memory: a century of consolidation. Science. 2000;287(5451):248-251. 